\title{
ANÁLISE DE FLUORESCÊNCIA E BIOENSAIOS COMO MÉTODO DE CARACTERIZAÇÃO DA REMOÇÃO DE MICROCISTINA POR ADSORÇÃO PELO BAGAÇO DE CANA
}

\author{
A.R. Almeida ${ }^{1}$, T. S. Copelli ${ }^{2}$ e T.A.Pagioro ${ }^{3}$ \\ ${ }^{1}$ Universidade Tecnológica Federal do Paraná, Programa de Pós-Graduação em Ciência e \\ Tecnologia Ambiental- PPGCTA, Mestranda \\ ${ }^{2}$ Universidade Tecnológica Federal do Paraná, Programa de Pós-Graduação em Ciência e \\ Tecnologia Ambiental- PPGCTA, Mestranda \\ ${ }^{3}$ Universidade Tecnológica Federal do Paraná, Programa de Pós-Graduação em Ciência e \\ Tecnologia Ambiental- PPGCTA, Professor \\ E-mail: alinealmeida@utfpr.edu.br
}

\begin{abstract}
RESUMO - O processo de tratamento de água para consumo humano, quando apresenta cianobactérias na água a ser tratada, pode promover lise dessas células e algumas espécies liberam toxinas, que, dependendo das concentrações dessas na água tratada ocasiona a possibilidade de óbito ao ser humano, pois o tratamento convencional não consegue retêlas facilmente. Diante disso, o presente trabalho avaliou o efeito da adsorção do bagaço de cana com filtro de areia sobre a capacidade de remoção de microcistina do cultivo da cianobactéria Microcystis aeruginosa. O uso do bagaço junto ao filtro de areia sugeriu uma possível alternativa, somado a alta disponibilidade e baixo custo desse subproduto, para retenção da microcistina. O monitoramento da toxina foi através da análise de fluorescência e bioensaios ecotoxicológicos com Daphnia magna que apresentaram uma possível viabilidade sem a demanda de recursos sofisticados ou técnicas muito apuradas para identificar a eficiência da remoção da microcistina.
\end{abstract}

\section{INTRODUÇÃO}

O crescimento das florações de cianobactéria ocorre principalmente devido a excesso de compostos fosfatados e nitrogenados, o Brasil por possuir alta produção agrícola faz o uso constante de fertilizantes, esses são ricos naqueles componentes que no solo podem atingir o lençol freático e favorecem a eutrofização da água (BRASIL, 2003). Assim como a descarga de esgoto doméstico e industrial sem tratamento também contribuem para esse crescimento.

O maior problema das florações de cianobactéria é que algumas espécies eliminam substâncias tóxicas para o meio ambiente; e dependendo da concentração e do tempo de contato com essas, podem levar tanto o animal como o homem a óbito; por exemplo, a morte de 70 pessoas em Caruaru, Pernambuco, durante tratamento de hemodiálise utilizando a água contaminada com toxina de cianobactéria (SANCHES et al.,2012; e CARVALHO et al., 2013). 
Quando a água a ser tratada para o abastecimento humano apresenta cianobactérias demanda tanto um maior custo operacional como um maior tempo para melhor eficiência no processo do tratamento, principalmente, nas etapas de coagulação, filtração e sedimentação. Tendo ainda, nas duas primeiras etapas citadas, o perigo de haver rompimento das células de cianobactéria e liberação de toxina para a água (GIJSBERTSEN-ABRAHAMSE et al., 2006). Mesmo assim, acontece dessas etapas não serem eficientes o suficiente; e ser necessário a adsorção com carvão ativado, o que gera um custo ainda maior para as estações de tratamento de água, já que a maior parte desse é importado (ALBUQUERQUE et al., 2008).

Os processos convencionais de tratamento da água não sendo eficientes para remover toxinas de cianobactérias da água, estimulam o desenvolvimento de pesquisas com o uso de coagulantes alternativos, filtros de membrana, processos oxidativos avançados; e uso de bioadsorventes para isso, visando a minimização de recursos não renováveis, de subprodutos e a sustentabilidade (MOLICA et al.,2002; GIJSBERTSEN-ABRAHAMSE et al., 2006; e BRASIL, 2003).

Verificando essa necessidade e aproveitando o bagaço de cana-de-açúcar, que constitui um subproduto de 291,3 kg por tonelada de cana processada (CONAB, 2013), para o Brasil cuja safra 2013/2014 foi de 658,8 milhões de tonelada (CONAB, 2014), permite a utilização desse recurso renovável, que já apresenta viabilidade de adsorção, como um potencial adsorvente sustentável para retenção de cianotoxina (MONACO et al., 2002; MOLICA et al., 2013).

A microcistina é uma toxina hepatotóxica produzida pela cianobactéria Microcystis e outras; e é uma das mais encontradas em mananciais de abastecimento, o limite máximo permitido dela na água potável é de $1,0 \mu \mathrm{g}$. L ${ }^{-1}$, conforme a Portaria 2914/2011 (BRASIL, 2011). Uma forma de analisá-la é através de testes de ecotoxicidade, que podem ser eficientes instrumentos de avaliação da qualidade da água e/ou sedimento (MENDONÇA, 2006). Esses bioensaios ecotoxicológicos ou testes de ecotoxicidade podem apresentar uma análise quantitativa e qualitativa dos efeitos tóxicos de diversos contaminantes sobre os organismos aquáticos (MONTEIRO, 2000).

Os bioensaios utilizando o microcrustáceo Daphnia magna são realizados através de ensaios normatizados pela NBR 12713:2009 (ABNT, 2009); e dividem-se em testes de toxicidade agudo e crônico, tendo como maior objetivo verificar os efeitos adversos causados por um algum composto tóxico (DAHMS et al., 2011).

Os testes agudos avaliam a dose ou a concentração de um agente tóxico que tenha capacidade de produzir efeitos aos organismos expostos a um período relativamente curto e 
possibilitam determinar a imobilidade ou a mortalidade a $50 \%$ da população por métodos estatísticos (RESTANI, 2011).

Como a quantificação das toxinas das cianobactérias depende de um aparato técnico especializado e equipamentos sofisticados, de modo a facilitar o monitoramento de toxicidade, este trabalho teve como objetivo avaliar a eficiência da análise por fluorescência e por bioensaios agudos com Daphnia magna para verificar a retenção de microcistina em filtro de areia com bagaço de cana.

\section{MATERIAIS E MÉTODOS}

O cultivo de cianobactéria da espécie Microcystis aeruginosa foi realizado com a cepa B005 cedida pelo Laboratório de Botânica da UFSCAR, que possui capacidade de produção de toxinas do tipo MC-LR em $98 \%$ do total. Manteve-se o cultivo sob aeração constante em meio ASM-01, temperatura entre 25 e $28^{\circ} \mathrm{C}$, até a fase exponencial de crescimento, quando o número de células atingiu o $10^{6}$ cel. $\mathrm{mL}^{-1}$, a contagem foi realizada em microscópio eletrônico utilizando câmara de Neubauer.

No décimo quarto dia, armazenou-se 4,5 L do cultivo em garrafa de polietileno, submetendo-o a três ciclos de congelamento e descongelamento, para que todas as células de cianobactéria sofressem lise de sua parede celular e liberassem toda microcistina ao meio de cultivo.

O bagaço de cana foi lavado com água destilada com três enxágues; em seguida foi mantido em estufa a $60^{\circ} \mathrm{C}$ por 4 horas. Triturou-se em liquidificador industrial na faixa de 2,80 a 4,00 mm de granulometria (MONACO et al., 2002).

Foram desenvolvidos seis suportes de filtro com altura de $14 \mathrm{~cm}$ em batelada, cada um filtrando $500 \mathrm{~mL}$ de cultivo; sendo três de filtro denominado AREIA que conteve apenas leito de areia média com faixa granulométrica entre $0,250 \mathrm{~mm}$ a $0,125 \mathrm{~mm}$ na altura de $80 \mathrm{~mm}$ e, numa área equivalente a $50 \mathrm{~cm}^{2}$ de filtração; e os três filtros denominados CA com proporção 1:1 de areia média com a mesma faixa granulométrica citada e bagaço, mantendo altura e área do anterior.

Os filtrados foram armazenados em volume de $50 \mathrm{~mL}$ para a análise no fluorescência e no HPLC (TECPAR); e $450 \mathrm{~mL}$ para teste de toxicidade com Daphnia magna. A análise no fluorescência foi realizada no equipamento modelo Cary Elipse (marca Varian), tendo como 
controle o meio de cultivo puro e sequencialmente as amostras denominadas AREIA e CA. Os parâmetros utilizados para comprimento de onda no fluorescência foram os citados no trabalho de ZIEGMANN et al. (2010), compreendendo o sinal de emissão entre $200 \mathrm{~nm}$ a $750 \mathrm{~nm}$; e passo de $5 \mathrm{~nm}$.

Os testes de toxicidade com Daphnia magna foram realizados, de acordo com a norma NBR 12713:2009 (ABNT, 2009), triplicatas em recipientes de $50 \mathrm{~mL}$ com $40 \mathrm{~mL}$ de amostra em cada um. Em cada recipiente foram colocados 20 organismos neonatos entre $6 \mathrm{~h}$ e $24 \mathrm{~h}$, nas diluições de $100 \%, 75 \%, 50 \%, 25 \%$ e $10 \%$. Da mesma forma foi utilizado um controle sem a presença de microcistinas apenas com água de diluição.

\section{RESULTADOS E DISCUSSÃO}

Na Tabela 1, apresentam-se os resultados obtidos pelo HPLC, laudo técnico fornecido pelo TECPAR (Centro de Análises e Ensaios Toxicológicos, Laboratório de Tecnologias Ambientais e Agronômicas do Estado do Paraná) para quantificação de toxina na amostra antes e após o tratamento de retenção de microcistina pela filtração:

Tabela 1- Dados quantitativos de microcistinas por HPLC (TECPAR, 2014)

\begin{tabular}{ccc}
\hline Amostra & $\begin{array}{c}\text { Média da Concentração de Microcistina MC-LR } \\
\left(\mu \mathrm{g} . \mathrm{L}^{-1}\right)\end{array}$ & $\begin{array}{c}\text { Desvio } \\
\text { Padrão }\end{array}$ \\
\hline Cultivo Puro & 89,0 & 0,50 \\
Areia & 2,42 & 0,36 \\
CA & 3,23 & 0,06 \\
\hline
\end{tabular}

Esses resultados foram utilizados como parâmetro para as análises de fluorescência e bioensaios com Daphnia magna, já que o método de quantificação de microcistina por HPLC é o mais eficiente atualmente. Verificando-se que houve retenção de microcistina em 97,3\% de eficiência com filtro composto só com areia média $(\mathrm{h}=80 \mathrm{~mm})$; e $96,4 \%$ para o filtro composto com areia média $(\mathrm{h}=40 \mathrm{~mm})$ e bagaço de cana $(\mathrm{h}=40 \mathrm{~mm})$.

As análises de fluorescência foram realizadas utilizando-se as médias das triplicadas dos dados de emissão das amostras para os comprimentos de onda; e os resultados foram apresentados na Figura 1, demonstrando que o sinal de incidência vai diminuindo conforme o comprimento de onda vai aumentando, com destaque ao intervalo entre 500 e $680 \mathrm{~nm}$ que equivale ao sinal de pigmentos da cianobactéria, conforme relata ZIEGMANN et al. (2010). No caso do tratamento de remoção de microcistina tanto para o filtro de areia como para o bagaço de cana ocorreu a diminuição do sinal comparado ao do cultivo puro. 


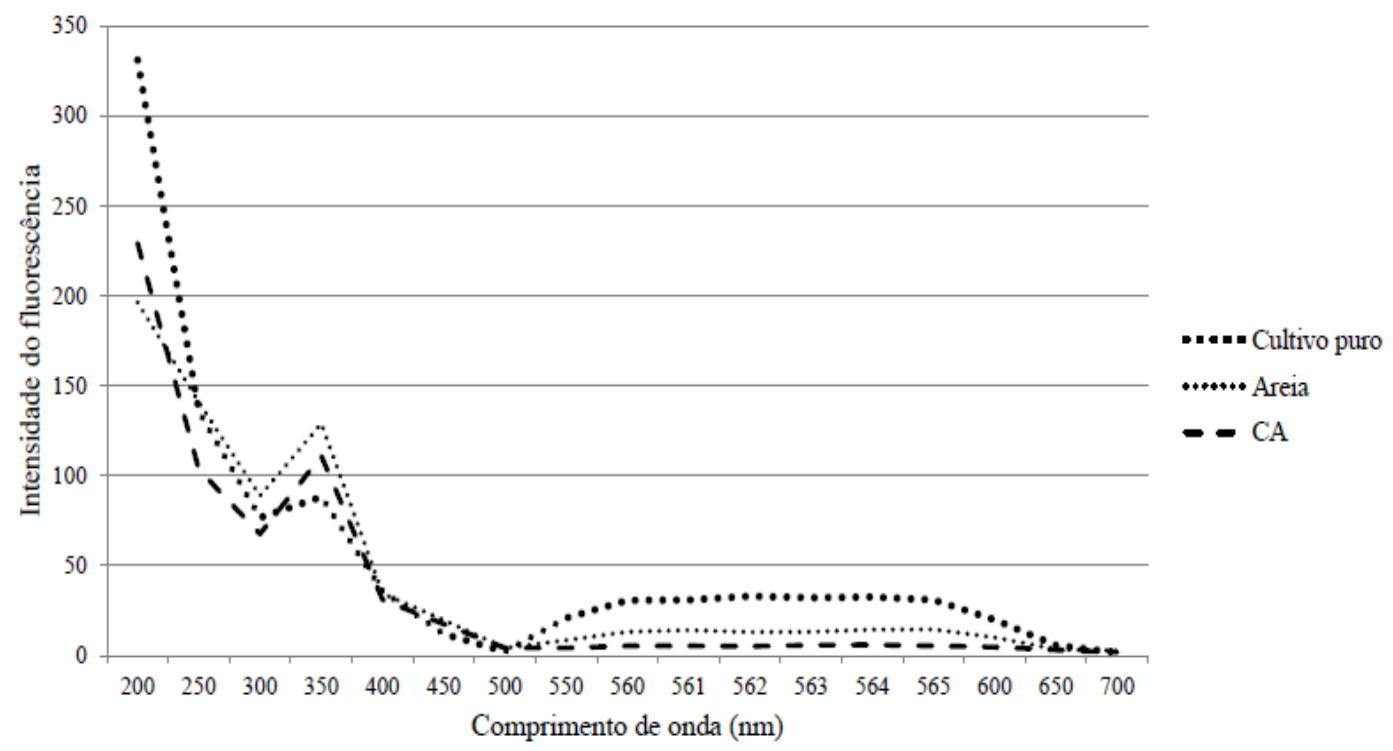

Figura 1- Decaimento de intensidade nas amostras no intervalo de 200 a $700 \mathrm{~nm}$ de comprimento de onda.

A $\mathrm{CE}_{50}$ para as amostras foi calculada através do programa Trimmed Spearman-Karber (HAMILTON et al., 1977) sendo de $34,87 \mu \mathrm{g} . \mathrm{L}^{-1}, 1,51 \mu \mathrm{g} . \mathrm{L}^{-1}$ e $1,46 \mu \mathrm{g} . \mathrm{L}^{-1}$ para as amostras cultivo puro, areia e CA respectivamente, conforme apresentado na Figura 2 abaixo.

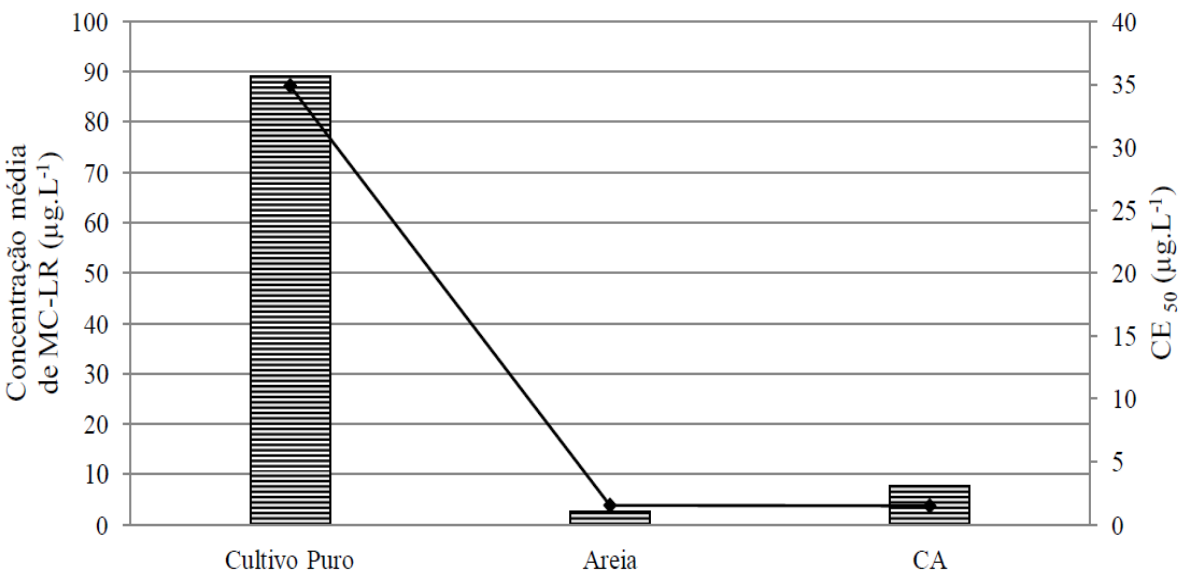

Figura 2- Concentração média de microcistina MC-LR e de Concentração efetiva $\left(\mathrm{CE}_{50}\right), 48 \mathrm{~h}$ para Daphnia magna em cada amostra.

A amostra tratada com o conjunto de filtro de areia e bagaço de cana continha uma menor quantidade de microcistina, porém, houve um percentual de imobilidade maior para essas amostras (95\% e 96,67\%) quando comparada com a amostra de cultivo puro $(91,67 \%$ e $90 \%$ ) para as diluições de $100 \%$ e $75 \%$, como apresentado abaixo pela Figura 3. 


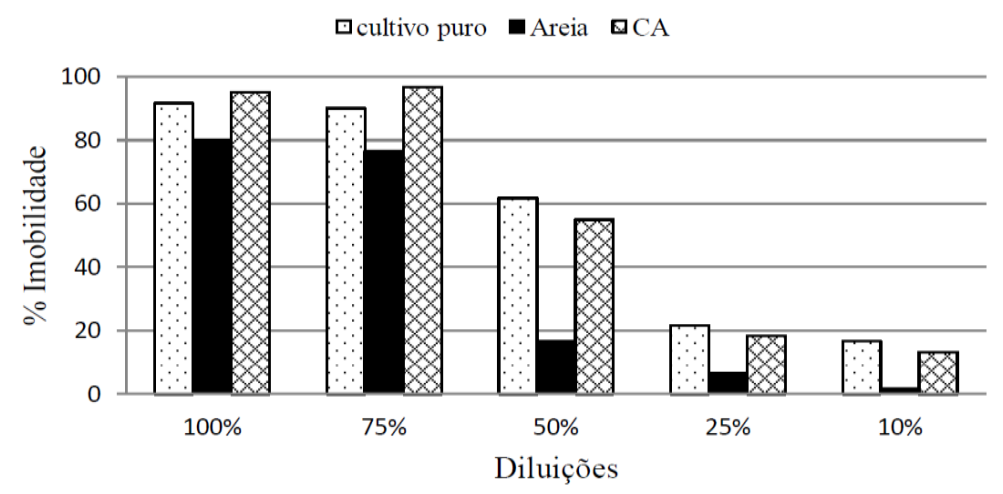

Figura 3- Percentagem de imobilidade de Daphnia magna após 48h de exposição às amostras de cultivo puro, cultivo após filtro de areia (Areia); e cultivo após conjunto de filtro de areia e bagaço de cana (CA).

As cianobactérias são responsáveis pela produção de polissacarídeos extracelulares (LOMBARDI; VIEIRA, 1998), que podem ser absorvidos pelos cladóceros (NOGUEIRA et al., 2005). A esses compostos bioativos também pode ser atribuído um efeito adverso, conforme Trabeau et al, (2004) que relacionou os efeitos adversos observados em Daphnia a polissacarídeos da cápsula de Microcystis.

Este trabalho não verificou os compostos acima mencionados, entretanto podemos indicar de acordo com Takenaka, (2007) que a presença desses metabólitos poderia aumentar a toxicidade dos extratos que os daphnídeos foram submetidos, com concentrações relativamente mais baixas de microcistinas. Sendo assim, pode-se afirmar que diversos fatores interferem na toxicidade e outras variantes também podem contribuir para que amostras com menor concentração de toxinas possam ser mais tóxicas que outras com concentrações mais elevadas (TAKENAKA, 2007).

\section{CONCLUSÃO}

As amostras contendo microcistinas, mesmo após o processo de adsorção ainda apresentou toxicidade, mesmo com concentrações mais baixas de microcistina. Esse fato pode ser atribuído a outros metabólitos secundários produzidos pela cianobactéria em questão que não foram analisadas neste trabalho.

Os resultados obtidos pela análise de fluorescência corresponderam à retenção de microcistina tanto pelo bagaço de cana como pelo filtro composto apenas com areia, trazendo a viabilidade dessa análise para monitoramento de microcistina, assim como a necessidade de se verificar tempo limite de adsorção e tamanho de leito diferente para uma ótima taxa de retenção da toxina. 


\section{REFERÊNCIAS}

ASSOCIAÇÃO BRASILEIRA DE NORMAS TÉCNICAS NBR12713: Ecotoxicologia aquática - Toxicidade aguda - Método de ensaio com Daphnia ssp (Crustacea, Cladocera). Rio de Janeiro, 2009.

AlbuQuerQue, E. C.J.; MÉNDEZ, M. O. COUTINHO, A.R.; FRANCO, T. T. A. Removal of cyanobacteria toxins from drinking water by adsorption on activated carbon fibers. Materials Research, v. 11, n.3, p. 371-380, 2008.

BRASIL. Cianobactérias tóxicas na água para consumo humano na saúde pública e processos de remoção em água para consumo humano. Brasília: Ministério da Saúde- Fundação Nacional da Saúde, p. 56, 2003.

BRASIL, Ministério da Saúde. Secretaria de Vigilância em Saúde. Coordenação Geral da Vigilância em Saúde Ambiental. Portaria n.2914, 2011.

CARVAlHO, M. C.; AGUJARO, L.F.; PIRES, D.A.; PICOLI, C. Manual de cianobactérias planctônicas: legislação, orientações para o monitoramento e aspectos ambientais. São Paulo: CETESB, 56 p., 2013.

CONAB. Perfil do Setor do Açúcar e do Álcool no Brasil Safra 2011/2012- Diretoria de Política Agrícola e Informações, Superintendência de Informações do Agronegócio. Responsáveis Técnicos: Ângelo Bressan Filho e Roberto Alves de Andrade. Brasília: CONAB, v.5, p.1-88, 2013.

CONAB. Acompanhamento da safra brasileira de cana-de-açúcar, Safra 2013/2014Quarto Levantamento. Brasília: CONAB, p. 1-14, abr., 2014.

DAHMS, H.U.; HAGIWARA, A.; LEE, J.S. Ecotoxicology, ecophysiology, and mechanistic studies with rotifers. Aquatic toxicology, v. 101, p. 1-12, 2011.

GILSBERTSEN-ABRAHAMSE, A.J., SCHMIDT, W. CHORUS, I.; HEIJMAN, S.G.J. Removal of cyanotoxins by ultrafiltration and nanofiltration. Journal of Membrane Science. v. 276, p. 252-259, 2006.

HAMILTON, M. A.; RUSSO, R. C \& THURRTON, R. B. Trimmed Spearman-Karber methods for estimating median letal concentration in toxicity bioassay. Environmental Science Technology. v. 11, p. 714-719, 1977.

LOMBARDI, A. T.; VIEIRA, A. A.H. Copper and lead complexation by high molecular weight compounds produced by Synura sp. (Chysophyceae) Phycologia. v. 37, p. 34-39, 1998. 
MENDONÇA, J. M. S. Avaliação da toxicidade de florações naturais e de cultura de cianobactérias: efeito dobre Ceriodaphnia silvestrii (Caldocera, Crustacea).Natal, 2006. 97 p. Dissertação (Mestrado em Bioecologia aquática). Centro de Biociências, Universidade Federal do Rio Grande do Norte.

MOLICA, R. J. R.; DUARTE, M. M. B.; AVELAR, F. P.; LIMA, N. M. F.; NEVES, C. C. L.; BARAUNA, O. S.; SILVA, P. W. S.; LEONIDIO, T. O. Adsorção de cianotoxinas em diferentes matrizes. In: BRASIL. $5^{\circ}$. Caderno de Pesquisa de Engenharia de Saúde Pública. $2^{\circ}$. Edição. Brasília: FUNASA, 2013, 166 p.

MONACO, P.A.L.; MATOS, A.T.; MARTINEZ, M.A.; JORDÃO, C.P. Eficiência de materiais orgânicos filtrantes no tratamento de águas residuárias da lavagem e despolpa dos frutos do cafeeiro. Engenharia na Agricultura, Viçosa, v. 10, n. 1-4, jan./dez., 2002.

MONTEIRO, N. J. C. Estudos da toxicidade da cepa de Microcystis aeruginosa RST 9501 da lagoa dos patos sobre cladócera. Porto Alegre, 2000. 197 p. Dissertação (Mestrado em Ecologia). Instituto de Biociências. Universidade Federal do Rio Grande do Sul.

NOGUEIRA, P.F. M.; MELÃO, M. G. G.; LOMBARDI, A. T; VIEIRA, A. A. H. The effects of Anabaena spitoides (Cyanophyceae) exopolysaccharide on copper toxicity to Simocephalus serralatus (Cladocera, Daphnidae). Freswater Biology. v. 50, p. 1560-1567, 2005.

RESTANI, G. C. Efeitos de cepas tóxicas e não tóxicas de Cilindrospermopsis raciborskii sobre aspectos do ciclo de vida de Daphnia laevis (Cladocera, Daphnidae). Itajuba, 2011. 67 p. Dissertação (Mestrado em Meio Ambiente e Recursos Hídricos). Universidade Federal de Itajubá.

SANCHES, S.M.; PRADO, E.L.; FERREIRA, I.M.; BRAGA, H.F.; VIEIRA, E.M. Presença da toxina microcistina em água, impactos na saúde pública e medidas de controle. Revista de Ciências Farmacêuticas Básica e Aplicada, v. 33, p. 181-187, 2012.

TRABEAU, M.; BRUHN-KEUP, R.; MCDERMOTT, C.; KEOMANY,M.; MILLSAPS, A.; EMERY, A.; STASIO JR, B. Midsummer decline of a Daphnia population attributed in part to cyanobacterial capsule production. Journal Plankton Research. v. 26, p. 942-961, 2004.

ZIEGMANN, M.; ALBERT, M.; MÜLLER, M.; FRIMMEL, F.H. Use of fluorescence fingerprints for estimation of bloom formation and toxin production of Microcystis aeruginosa. Water Research, v. 44, p. 195-204, 2010.

TAKENAKA, R. A.; DELLAMANO-OLIVEIRA, M.J.; ROCHA, O. Toxicidade de extratos de florações de cianobactérias de reservatórios do rio Tietê, SP, aos dafinídeos Ceriodaphnia dúbia e Ceriodaphnia silvestrii (Cladocera, Crustacea). Journal of the Brazilian Society of Ecotoxicology. v. 2, p. 147-156, 2007. 